\title{
MULTIPLICATION MODULES WHOSE ENDOMORPHISM RINGS ARE INTEGRAL DOMAINS
}

\author{
SAng Cheol Lee
}

\begin{abstract}
In this paper, several properties of endomorphism rings of modules are investigated. A multiplication module $M$ over a commutative ring $R$ induces a commutative ring $M^{*}$ of endomorphisms of $M$ and hence the relation between the prime (maximal) submodules of $M$ and the prime (maximal) ideals of $M^{*}$ can be found. In particular, two classes of ideals of $M^{*}$ are discussed in this paper: one is of the form $G_{M^{*}}(M, N)=$ $\left\{f \in M^{*} \mid f(M) \subseteq N\right\}$ and the other is of the form $G_{M^{*}}(N, 0)=\{f \in$ $\left.M^{*} \mid f(N)=0\right\}$ for a submodule $N$ of $M$.
\end{abstract}

\section{Introduction}

Throughout this paper, unless otherwise specified, we shall assume that all rings are associative with identity and all modules are unitary left modules.

Let $R$ be a ring and let $M$ be an $R$-module. Then the set of all $R$ homomorphisms from $M$ into itself can be given the structure of a ring. We call this ring the ring of endomorphisms of $M$ and denote this by $M^{*}$.

Let $L$ and $N$ be any two submodules of $M$. Then the set

$$
\left\{f \in M^{*} \mid f(L) \subseteq N\right\}
$$

will be considered. This set becomes an additive subgroup of the group $\left(M^{*},+\right)$. So, we will denote this subgroup by $G_{M^{*}}(L, N)$.

If we make different choices of $L$ and $N$, then $G_{M^{*}}(L, N)$ has different algebraic structures. There are four cases to consider:

$$
\text { (1) } L \supseteq N, \quad \text { (2) } L \subseteq N, \quad \text { (3) } L \nsupseteq N, \quad \text { (4) } L \nsubseteq N .
$$

In case of (1), $G_{M^{*}}(L, N)$ is a subring of the ring $M^{*}$. In particular, $G_{M^{*}}(0,0)=M^{*}, G_{M^{*}}(M, 0)=0$, and $G_{M^{*}}(M, M)=M^{*}$.

As special cases of $(2), G_{M^{*}}(M, M)=M^{*}$ and for any submodule $N$ of $M$, $G_{M^{*}}(0, N)=M^{*}$.

Received April 10, 2009.

2000 Mathematics Subject Classification. 13C05, 13C10, 13C11.

Key words and phrases. multiplication module, semi-injective module, self-cogenerated module, tight closed submodule and closed submodule.

This paper was (partially) supported by the CBNU funds for overseas research, 2006 (OR-2006-XX). 
In cases of (3) and (4), we do not know the further algebraic structure of $G_{M^{*}}(L, N)$.

Now, let $N$ be a submodule of $M$. Then we get $M \supseteq N \supseteq 0$. So, by (1) we get three subrings of $M^{*}: G_{M^{*}}(M, N), G_{M^{*}}(N, N)$, and $G_{M^{*}}(N, 0)$. We will discuss about these three subrings of $M^{*}$. Of course, they have inclusion relation as follows:

$$
G_{M^{*}}(N, 0) \subseteq G_{M^{*}}(N, N) \supseteq G_{M^{*}}(M, N) .
$$

\section{Endomorphism rings}

Let $R$ be a ring. Let $M$ be an $R$-module. Define a ring homomorphism $\varphi: R \rightarrow M^{*}$ to be $\varphi(r)=\varphi_{r}: M \rightarrow M$ with $\varphi_{r}(x)=r x$. Then

$$
R / \operatorname{Ann}_{R}(M) \cong \operatorname{Im}(\varphi) \subseteq M^{*} .
$$

The $\varphi$ may not be injective. The example of this is given below.

Example 1.1. Take $R=\mathbb{Z}, M=\mathbb{Z} / 2 \mathbb{Z}$. Then $2 \in \operatorname{Ann}_{R}(M)$.

When $M$ is a faithful $R$-module, however, $\varphi$ is injective. If $V$ is a non-zero vector space over a field $F$, then $V$ is faithful over $F$. So, $\varphi: F \rightarrow V^{*}$ is injective. Hence, $F$ can be embedded in $V^{*}$. If $M$ is a non-zero free module over a commutative ring with identity with finite rank, then $M$ is also faithful over $R$. So, $\varphi: R \rightarrow M^{*}$ is injective. Hence, $R$ can be embedded in $M^{*}$.

Proposition 1.2. Let $R$ be a ring. Let $M$ be an $R$-module. If $\varphi: R \rightarrow M^{*}$ is surjective and $M^{*}$ is a projective $R$-module with rank 1 , then $\varphi$ is injective and hence $R \cong M^{*}$.

Proof. The following exact sequence

$$
0 \longrightarrow \operatorname{Ker}(\varphi) \longrightarrow R \stackrel{\varphi}{\longrightarrow} M^{*} \longrightarrow 0
$$

splits. So, $R=\operatorname{Ker}(\varphi) \oplus M^{*}$. Let $\mathfrak{p}$ be any element of $\operatorname{Spec}(R)$. Then $R_{\mathfrak{p}}=$ $\operatorname{Ker}(\varphi)_{\mathfrak{p}} \oplus M_{\mathfrak{p}}^{*}$. Since $M_{\mathfrak{p}}^{*}$ is $R_{\mathfrak{p}}$-free with rank 1 , we have $\operatorname{Ker}(\varphi)_{\mathfrak{p}}=0$. This shows that $\operatorname{Ker}(\varphi)=0$. Hence $\varphi$ is injective.

While discussing projective modules [9] with Professor Satya Mandal, we could see incidently that every projective module with positive rank over a reduced Noetherian ring is faithful.

Lemma 1.3. If $R$ is a reduced Noetherian ring, then every finitely generated projective $R$-module with positive rank is faithful.

Proof. Let $R$ be a reduced Noetherian ring and let $P$ be any finitely generated projective $R$-module with positive rank. Let $\mathfrak{p}$ be any minimal prime ideal of $R$. Let $x$ be any element of $\operatorname{Ann}_{R} P$. Then $x P=0$, and so $(x / 1) P_{\mathfrak{p}}=0 . P_{\mathfrak{p}}$ is a non-zero free $R_{\mathfrak{p}}$-module. Notice that every non-zero free module with finite rank is faithful. Then $x / 1=0$, so there exists an element $s \in R \backslash \mathfrak{p}$ such that $s x=0 . \quad s x=0 \in \mathfrak{p}$. Hence, $x \in \mathfrak{p}$. This shows that $\operatorname{Ann}_{R} P \subseteq \mathfrak{p}$. Thus, $\operatorname{Ann}_{R} P \subseteq \cap_{\mathfrak{p} \in \operatorname{Min}(R)} \mathfrak{p}=\sqrt{0}=0$. Therefore, $\operatorname{Ann}_{R} P=0$. 
Let $R$ be a commutative ring with identity and let $M$ be an $R$-module. Then $M$ is called a multiplication module if for every submodule $N$ of $M$ there exists an ideal $I$ of $R$ such that $N=I M$. If $R$ is a commutative ring with identity, then $R$ is a multiplication module over $R$. If $V$ is a vector space over a field $k$ and if the dimension of $V$ over $k$ is greater than 1 , then $V$ is not a multiplication module over $k$. For otherwise, for a subspace $W$ of $V$ with $\operatorname{dim}_{k}(W)=1$, there exists an ideal $I$ of $k$ such that $W=I V$. Since the only ideals of the field $k$ are 0 and $k$ itself, we have $W=0$ or $W=V$. This is a contradiction.

Let $R$ be a ring and let $M$ be an $R$-module. Let $f \in M^{*}$. A submodule $N$ of $M$ such that $f(N) \subseteq N$ is called $f$-stable or $f$-invariant. Further, recall that a submodule $N$ of $M$ is called fully invariant if for every $f \in M^{*}, N$ is $f$-invariant, or equivalently, if $M^{*}=G_{M^{*}}(N, N)$.

Let $R$ be a commutative ring with identity and let $M$ be a multiplication module. Let $N$ be any submodule of $M$. Then there exists an ideal $I$ of $R$ such that $N=I M$. Now, let $f$ be any element of $M^{*}$. Then

$$
f(N)=f(I M)=I f(M) \subseteq I M=N .
$$

Hence $N$ is $f$-invariant. Therefore $N$ is fully invariant. We have proved the following.

Lemma 1.4 ([6, Proposition 7] and [4, Lemma 1]). If $M$ is a multiplication module over a commutative ring with identity, then every submodule of $M$ is fully invariant.

Let $R$ be a commutative ring with identity. For every $R$-module $M, M^{*}$ is a ring with identity. Assume further that $M$ is a multiplication module. Let $m$ be any element of $M$. Then by Lemma $1.4, R m$ is fully invariant. Let $f$ be any element of $M^{*}$. Then $f(m) \in f(R m) \subseteq R m$. There exists an element $r \in R$ such that $f(m)=r m$. If $g$ is any element of $M^{*}$, then by a similar proof we can find an element $s \in R$ such that $g(m)=s m$. Hence

$$
(f g)(m)=s(r m)=(s r) m=(r s) m=r(s m)=(g f)(m) .
$$

Hence $f g=g f$. Therefore, $M^{*}$ is a commutative ring with identity (see [3, Lemma 2]).

Let $R$ be a ring. An element $r$ of $R$ is called a zero-divisor if there exists a non-zero element $s$ in $R$ such that $r s=0$. From now on we denote the set of all zero-divisors of a ring $R$ by $Z(R)$. A commutative $\operatorname{ring} R$ with identity is called an integral domain if $Z(R)=0$.

Theorem 1.5. If $M$ is a faithful multiplication module over an integral domain, then $M^{*}$ is an integral domain.

Proof. $M^{*}$ is a commutative ring with identity. So, it is sufficient to prove: if $f g=0$, where $f, g \in M^{*}$, then either $f$ or $g$ is zero.

There are ideals $I, J$ of $R$ such that $f(M)=I M, g(M)=J M$. Then $0=(f g)(M)=J(I M)=(J I) M=(I J) M$, so $I J \subseteq \operatorname{Ann}_{R}(M)=0$. Hence, 
$I J=0$. Since $R$ is an integral domain, either $I$ or $J$ is zero. If $I=0$, then $f(M)=0$. If $J=0$, then $g(M)=0$. Hence, either $f$ or $g$ is zero.

Every integral domain is reduced. Hence the next result follows from Lemma 1.3 and Theorem 1.5.

Corollary 1.6. Let $R$ be a Noetherian domain. If $P$ is a finitely generated projective multiplication $R$-module with positive rank, then $P^{*}$ is an integral domain. $2.4]$.

The following result was motivated by $[12$, Proposition 1.2] and [8, Theorem

Lemma 1.7. Let $R$ be a commutative ring with identity. Let $M$ be a finitely generated $R$-module.

(1) If $f: M \rightarrow M$ is an epimorphism, then $f$ satisfies a polynomial of the form

$$
1+a_{1} X+a_{2} X^{2}+\cdots+a_{n} X^{n}
$$

where the $a_{i}$ are in $R$.

(2) If $f: M \rightarrow M$ is an epimorphism, then $f$ is a monomorphism.

Let $R$ be a commutative ring with identity. Let $E$ be an $R$-module. An element $e$ of $E$ is said to be divisible if, for every $r$ of $R \backslash Z(R)$, there exists $e^{\prime} \in E$ such that $e=r e^{\prime}$. If every element of $E$ is divisible, then $E$ is said to be a divisible module. Alternatively, $E$ is divisible if $E=r E$ whenever $r$ is an element of $R \backslash Z(R)$.

Let $R$ be an integral domain. If $E$ is a non-zero divisible $R$-module, then the ring homomorphism $\varphi: R \rightarrow E^{*}$ which was discussed in the paragraph just prior to Example 1.1 is injective. In other words, if multiplication by $r$ is zero, then $r$, as an element of $R$, is zero.

Theorem 1.8. If an integral domain admits a non-zero finitely generated injective module, then it is a field.

Proof. Let $R$ be an integral domain and let $E$ be a non-zero finitely generated injective module. Then $E$ is divisible by [11, Proposition 2.6]. Let $r$ be a non-zero element of $R$. Then $r E=E$. Hence, multiplication by $r$ is an epimorphism. By Theorem 1.7(1), $r$ satisfies a polynomial of the form

$$
1+a_{1} X+a_{2} X^{2}+\cdots+a_{n} X^{n},
$$

where the $a_{i}$ are in $R$. Hence,

$$
1+a_{1} r+a_{2} r^{2}+\cdots+a_{n} r^{n}=0 .
$$

This means that $1+a_{1} r+a_{2} r^{2}+\cdots+a_{n} r^{n}$, as an element of $E^{*}$, is zero. By the argument just prior to Theorem 1.8, $1+a_{1} r+a_{2} r^{2}+\cdots+a_{n} r^{n}$, as an element of $R$, is zero. Hence, $\left(-a_{1}-a_{2} r-\cdots-a_{n} r^{n-1}\right) r=1$. Therefore, $r$ is invertible. 
Corollary 1.9. If $E$ is a non-zero finitely generated injective module over an integral domain, $E^{*}$ is a field.

Proof. By [8, Theorem 2.1, p. 7], $E^{*}$ is integral over $R$. By Theorem $1.8, R$ is a field. Hence, by $[8$, Lemma 1, p. 66$], E^{*}$ is a field.

Let $R$ be a ring and let $M$ be an $R$-module. Then we can give $M$ an $M^{*}$ module structure as follows:

$$
f \cdot m=f(m),
$$

where $f \in M^{*}$ and $m \in M$.

Let $f$ be any element of $\operatorname{Ann}_{M^{*}}(M)$. Then $f(M)=f \cdot M=0$ and hence $f=0$. This shows that $\operatorname{Ann}_{M^{*}}(M)=0$. Hence every $R$-module $M$ can be viewed as a faithful $M^{*}$-module.

Lemma 1.10. Let $R$ be a commutative ring with identity. Let $M$ be an $R$ module. If $M$ is a multiplication module over $R$, then $M$ is a faithful multiplication module over $M^{*}$.

Proof. Let $M$ be a multiplication module over $R$. Let $N$ be any $M^{*}$-submodule of the $M^{*}$-module $M$. Then for any $r \in R$ and for any $n \in N, r n=\varphi_{r}(n)=$ $\varphi_{r} . n \in N$. Hence, $N$ is an $R$-submodule of $M$. There exists an ideal $I$ of $R$ such that $N=I M$. Let $\varphi_{I}=\left\{\varphi_{r} \mid r \in I\right\}$. Then $\varphi_{I} M^{*}$ is an ideal of $M^{*}$ (generated by $\varphi_{I} \subseteq M^{*}$ ) and

$$
\left(\varphi_{I} M^{*}\right) \cdot M=\varphi_{I} \cdot\left(M^{*} \cdot M\right)=\varphi_{I} \cdot M=\varphi_{I}(M)=I M=N .
$$

Hence, $M$ is also a multiplication module over $M^{*}$.

Every vector space over a field is injective. Hence the next result follows from Corollary 1.9 and Lemma 1.10.

Corollary 1.11. If E is a non-zero, finitely generated, injective, multiplication module over an integral domain, then it is a non-zero, faithful, finitely generated, injective, multiplication module over the field $E^{*}$.

\section{2. $G_{M^{*}}(M, N)$}

Let $N$ be any submodule of $M$. The subring $G_{M^{*}}(M, N)$ of $M^{*}$ will be considered. This is a right ideal of the ring $M^{*}$. However, $G_{M^{*}}(M, N)$ is not always a left ideal of $M^{*}$. The example of this is given below.

Example 2.1. Let $R$ be a ring with identity $\neq 0$ and let $M$ be a free $R$ module with rank 2 . Let $\left\{e_{1}, e_{2}\right\}$ be an $R$-free basis for $M$. Consider the following submodule of $M$ :

$$
\nabla=\left\{a e_{1}+a e_{2} \mid a \in R\right\} .
$$

Then $G_{M^{*}}(M, \nabla)$ is not a left ideal of $M^{*}$. In fact, define a map $f: M \rightarrow M$ by $f\left(a e_{1}+b e_{2}\right)=a e_{1}+a e_{2}$, where $a, b \in R$. Then $f \in G_{M^{*}}(M, \nabla)$. Now, 
define a map $\alpha: M \rightarrow M$ by $\alpha\left(a e_{1}+b e_{2}\right)=a e_{1}$, where $a, b \in R$. Then $\alpha \in M^{*}$. Further, $\alpha f \notin G_{M^{*}}(M, \nabla)$. For otherwise,

$$
\nabla \ni(\alpha f)\left(e_{1}\right)=\alpha\left(e_{1}+e_{2}\right)=e_{1} .
$$

This is a contradiction. Therefore $G_{M^{*}}(M, \nabla)$ is not a left ideal of $M^{*}$.

Lemma 2.2. Let $R$ be a ring and let $M$ be an $R$-module. Then for every fully invariant submodule $N$ of $M, G_{M^{*}}(M, N)$ is a two-sided ideal of $M^{*}$.

Proof. We have already known that $G_{M^{*}}(M, N)$ is a right ideal of $M^{*}$. Now, let $\alpha \in M^{*}$ and $f \in G_{M^{*}}(M, N)$. Then $(\alpha f)(M) \subseteq \alpha(N) \subseteq N$. Hence $\alpha f \in G_{M^{*}}(M, N)$.

If $M$ is a multiplication module over a commutative ring with identity, then for every submodule $N$ of $M, G_{M^{*}}(M, N)$ is a two-sided ideal of $M^{*}$ by Lemma 1.4 .

Theorem 2.3. Let $R$ be a commutative ring with identity. Let $M$ be an $R$ module. Assume that $M$ is a multiplication $R$-module. Then $P$ is a prime submodule of $M$ if and only if $G_{M^{*}}(M, P)$ is a prime ideal of $M^{*}$.

Proof. Recall that $M^{*}$ is a commutative ring with identity.

Assume that $P$ is a prime submodule of $M$. Suppose $G_{M^{*}}(M, P)=M^{*}$. Consider the identity map $1_{M}: M \rightarrow M$. Then $1_{M} \in M^{*}=G_{M^{*}}(M, P)$, so $M=1_{M}(M) \subseteq P$. Hence $P=M$, which implies a contradiction. Hence $G_{M^{*}}(M, P) \neq M^{*}$.

Now, assume that $f g \in G_{M^{*}}(M, P)$, where $f, g \in M^{*}$. Then since $M$ is a multiplication $R$-module, there are ideals $I$ and $J$ of $R$ such that $f(M)=I M$ and $g(M)=J M$. So,

$$
(I J) M=(J I) M=J(I M)=J(f(M))=f(J M)=f(g(M)) \subseteq P .
$$

This implies that $I J \subseteq\left(P:_{R} M\right)$. Since $P$ is a prime submodule of $M$, it is well-known $([7$, p. 2$])$ that $\left(P:_{R} M\right)$ is a prime ideal of $R$. Hence

$$
I \subseteq\left(P:_{R} M\right) \text { or } J \subseteq\left(P:_{R} M\right) .
$$

Assume that $I \subseteq\left(P:_{R} M\right)$. Then $f(M)=I M \subseteq P$, so $f \in G_{M^{*}}(M, P)$. Or, assume that $J \subseteq\left(P:_{R} M\right)$. Then $g(M)=J M \subseteq P$, so $g \in G_{M^{*}}(M, P)$.

Therefore, $G_{M^{*}}(M, P)$ is a prime ideal of $M^{*}$.

Conversely, assume that $G_{M^{*}}(M, P)$ is a prime ideal of $M^{*}$. Suppose that $P=M$. Then $G_{M^{*}}(M, P)=G_{M^{*}}(M, M)=M^{*}$. This is a contradiction. Hence $P \neq M$.

Assume that $r m \in P$, where $r \in R$ and $m \in M$. Since $M$ is a multiplication $R$-module, there exists an ideal $I$ of $R$ such that $R m=I M$. So,

$$
(r I) M=r(I M)=(r R) m \subseteq P .
$$

Consider the ring homomorphism $\varphi: R \rightarrow M^{*}$ which was discussed in the paragraph just prior to Example 1.1. Since $G_{M^{*}}(M, P)$ is a prime ideal of $M^{*}$, 
it follows that $\varphi^{-1}\left(G_{M^{*}}(M, P)\right)$ is a prime ideal of $R$. Further, notice that $\varphi_{r I}(M)=(r I) M \subseteq P$. Then $\varphi(r I)=\varphi_{r I} \subseteq G_{M^{*}}(M, P)$. This implies that $r I \subseteq \varphi^{-1}\left(G_{M^{*}}(M, P)\right)$. Hence $r \in \varphi^{-1}\left(G_{M^{*}}(M, P)\right)$ or $I \subseteq \varphi^{-1}\left(G_{M^{*}}(M, P)\right)$. Assume that $r \in \varphi^{-1}\left(G_{M^{*}}(M, P)\right)$. Then $\varphi(r) \in G_{M^{*}}(M, P)$, so $r M=$ $\varphi_{r}(M) \subseteq P$. Hence $r \in(P: R M)$. Or, assume that $I \subseteq \varphi^{-1}\left(G_{M^{*}}(M, P)\right)$. Then $\varphi(I) \subseteq G_{M^{*}}(M, P)$, so $R m=I M=\varphi_{I}(M) \subseteq P$. Hence $m \in P$.

Therefore, $P$ is a prime submodule of $M$.

Lemma 2.4. Let $R$ be a commutative ring with identity and let $M$ be an $R$ module. If $M$ is a multiplication $R$-module, then for every submodule $N$ of $M$, $N=\sum_{f \in G_{M^{*}}(M, N)} f(M)$.

Proof. It is obvious that $\sum_{f \in G_{M^{*}}(M, N)} f(M) \subseteq N$.

Conversely, let $x$ be any element of $N$. Since $M$ is a multiplication $R$ module, there exists an ideal $I$ of $R$ such that $R x=I M$. Further, there are $a_{1}, a_{2}, \ldots, a_{r} \in I$ and $m_{1}, m_{2}, \ldots, m_{r} \in M$ such that $x=a_{1} m_{1}+a_{2} m_{2}+$ $\cdots+a_{r} m_{r}$. Let $\varphi: R \rightarrow M^{*}$ be as before. Then for each $i \in\{1,2, \ldots, r\}$, $\varphi_{a_{i}}(M)=a_{i} M \subseteq I M=R x \subseteq N$ and hence $\varphi_{a_{i}} \in G_{M^{*}}(M, N)$. Hence

$$
\begin{aligned}
x & =a_{1} m_{1}+a_{2} m_{2}+\cdots+a_{r} m_{r} \\
& \in \varphi_{a_{1}}(M)+\varphi_{a_{2}}(M)+\cdots+\varphi_{a_{r}}(M) \\
& \subseteq \sum_{f \in G_{M^{*}}(M, N)} f(M) .
\end{aligned}
$$

Hence $N \subseteq \sum_{f \in G_{M^{*}}(M, N)} f(M)$. Therefore $N=\sum_{f \in G_{M^{*}}(M, N)} f(M)$.

Consider the ring homomorphism $\varphi: R \rightarrow M^{*}$ which was discussed in the paragraph just prior to Example 1.1. $\varphi^{-1}\left(G_{M^{*}}(M, N)\right)$ will be denoted by $G_{M^{*}}(M, N) \cap R$. Then we have the following result.

Proposition 2.5. For every submodule $N$ of an $R$-module $M$,

$$
\left(N:_{R} M\right)=G_{M^{*}}(M, N) \cap R .
$$

Let $M$ be a multiplication $R$-module and let $N$ be any submodule of $M$. Then there exists an ideal $I$ of $R$ such that $N=I M$. This implies that $\left.I \subseteq N:_{R} M\right) M$. Hence $N=I M \subseteq\left(N:_{R} M\right) M$. Also, $\left(N:_{R} M\right) M \subseteq N$, which is clear from the definition. Hence $N=\left(N:_{R} M\right) M$. This is useful in the proof of the following result.

Theorem 2.6. Let $R$ be a commutative ring with an identity. Let $M$ be a finitely generated multiplication $R$-module. Then a submodule $N$ of $M$ is maximal if and only if $\left(N:_{R} M\right)$ is a maximal ideal of $R$.

Proof. Let $N$ be a maximal submodule of $M$. Assume that $J$ is an ideal of $R$ such that $\left(N:_{R} M\right) \subseteq J \subseteq R$. Since $M$ is a multiplication $R$-module, it follows from the above argument that

$$
N=\left(N:_{R} M\right) M \subseteq J M \subseteq M .
$$


By the maximality of $N$, either $J M=N$ or $J M=M$. Assume that $J M=N$. Then $J \subseteq\left(N:_{R} M\right)$. Hence $J=\left(N:_{R} M\right)$. Or, assume that $J M=M$. By the Nakayama Lemma, there exists an element $a \in J$ such that $(1-a) M=0$. So, $(1-a) M=0 \subseteq N$. This implies that $1-a \in\left(N:_{R} M\right) \subseteq J$. Hence $1=a+(1-a) \in J$. Hence $J=R$. This shows that $\left(N:_{R} M\right)$ is a maximal ideal of $R$.

Conversely, assume that $\left(N:_{R} M\right)$ is a maximal ideal of $R$. Let $A$ be a submodule of $M$ such that $N \subseteq A \subseteq M$. Then

$$
\left(N:_{R} M\right) \subseteq\left(A:_{R} M\right) \subseteq R .
$$

By the maximality of $\left(N:_{R} M\right)$, either $\left(A:_{R} M\right)=\left(N:_{R} M\right)$ or $\left(A:_{R} M\right)=$ $R$. Assume that $\left(A:_{R} M\right)=\left(N:_{R} M\right)$. Since $M$ is a multiplication module, we have $A=\left(A:_{R} M\right) M=\left(N:_{R} M\right) M=N$. Or, if $\left(A:_{R} M\right)=R$, then $M=A$. This shows that $N$ is a maximal submodule of $M$.

We have already known that if $R$ is a commutative ring with identity and $M$ is a multiplication module over $R$, then $M^{*}$ is a commutative ring with identity. If $M$, as an $R$-module, is finitely generated, then $M$, as an $M^{*}$-module, is also finitely generated. Compare the following result with Theorem 2.3.

Corollary 2.7. Let $R$ be a commutative ring with identity. Let $M$ be a finitely generated multiplication module over $R$ and let $N$ be any submodule of $M$. Then $N$ is a maximal $M^{*}$-submodule of the $M^{*}$-module $M$ if and only if $G_{M^{*}}(M, N)$ is a maximal ideal of $M^{*}$.

Proof. Note that $G_{M^{*}}(M, N)=\left(N:_{M^{*}} M\right)$. Then it suffices to prove that $N$ is a maximal $M^{*}$-submodule of the $M^{*}$-module $M$ if and only if $\left(N:_{M^{*}} M\right)$ is a maximal ideal of $M^{*}$. Use [5, Theorem 3.1, p. 768] to prove the 'only if part'. The remainder of the proof is almost the same as that of Theorem 2.6.

\section{3. $G_{M^{*}}(N, 0)$}

Let $R$ be ring and let $N$ be a submodule of $M$. Then $G_{M^{*}}(N, 0)$ is a left ideal of $M^{*}$. However, this is not a right ideal of $M^{*}$. The example of this is given below.

Example 3.1. Use the same notation as in Example 1.1. Define a map $g$ : $M \rightarrow M$ by $g\left(a e_{1}+b e_{2}\right)=(a-b) e_{1}$, where $a, b \in R$. Then $g \in G_{M^{*}}(\nabla, 0)$. Further,

$$
(g \alpha)\left(e_{1}+e_{2}\right)=g\left(\alpha\left(e_{1}+e_{2}\right)\right)=g\left(e_{1}\right)=e_{1} \neq 0 .
$$

Hence $g \alpha \notin G_{M^{*}}(\nabla, 0)$. Hence $G_{M^{*}}(\nabla, 0)$ is not a right ideal of $M^{*}$.

Compare the following lemma with Lemma 2.2.

Lemma 3.2. Let $R$ be a ring with identity and let $M$ be an $R$-module. Then for every fully invariant submodule $N$ of $M, G_{M^{*}}(N, 0)$ is a two-sided ideal of $M^{*}$ 
Proof. We have already known that $G_{M^{*}}(N, 0)$ is a left ideal of $M^{*}$. Now, let $\alpha \in M^{*}$ and $f \in G_{M^{*}}(N, 0)$. Then $(f \alpha)(N) \subseteq f(N)=0$. Hence $f \alpha \in$ $G_{M^{*}}(N, 0)$.

If $M$ is a multiplication module over a commutative ring with identity, then for every submodule $N$ of $M, G_{M^{*}}(N, 0)$ is a two-sided ideal of $M^{*}$.

Let $R$ be a ring. Let $M$ be an $R$-module and let $N$ be a submodule of $M$. Then for each $f \in G_{M^{*}}(N, 0), \operatorname{Ker}(f)$ contains $N$. Hence

$$
\cap_{f \in G_{M^{*}}(N, 0)} \operatorname{Ker}(f) \supseteq N \text {. }
$$

A submodule $N$ of an $R$-module $M$ is called to be tight closed if

$$
\cap_{f \in G_{M^{*}}(N, 0)} \operatorname{Ker}(f)=N .
$$

In papers [1] and [2], the name of the submodule in the definition was a "closed submodule", however we call it to be a tight closed submodule to avoid confusion with the name in [10]. Moreover, in view of the following Proposition 3.4 , it seems like to be reasonable for us to call the submodule a tight closed submodule.

Proposition 3.3. Let $R$ be a ring and let $M$ be an $R$-module. Let $N$ be a submodule of $M$. If there exists an element $f \in M^{*}$ such that $\operatorname{Ker}(f)=N$, then $N$ is tight closed.

Proof. Assume that there exists an element $f \in M^{*}$ such that $\operatorname{Ker}(f)=N$. Then $N \subseteq \cap_{g \in G_{M^{*}}(N, 0)} \operatorname{Ker}(g) \subseteq \operatorname{Ker}(f)=N$. Hence $\cap_{g \in G_{M^{*}}(N, 0)} \operatorname{Ker}(g)=N$. Therefore $N$ is tight closed.

Let $R$ be a ring and let $M$ be an $R$-module. Then $\operatorname{Ker}\left(1_{M}\right)=0$ and $\operatorname{Ker}\left(0_{M}\right)=M$. Hence, by Proposition 3.3, the zero submodule of $M$ and $M$ itself are tight closed and for any $f \in M^{*}, \operatorname{Ker}(f)$ is tight closed.

Let $V$ be a finite-dimensional vector space over a field. Let $W$ be any subspace of $V$. Then there exists a subspace $W^{\prime}$ of $V$ such that $V=W \oplus W^{\prime}$. So, we can define a map $f: V \rightarrow V$ such that $\left.f\right|_{W}=0$ and $\left.f\right|_{W^{\prime}}=1_{W^{\prime}}$. Then $f \in V^{*}$ and $\operatorname{Ker}(f)=W$. Hence $W$ is tight closed in $V$. Therefore every subspace of a finite-dimensional vector space $V$ over a field is tight closed in $V$.

Now, let $A$ be an algebra over a field $k$. Let $P$ be a finitely generated projective $A$-module. Then there exists an $A$-module $Q$ and an integer $n$ such that $P \oplus Q=A^{n}$. So, we can define a map $f: A^{n} \rightarrow A^{n}$ such that $\left.f\right|_{P}=0$ and $\left.f\right|_{Q}=1_{Q}$. Then $f \in\left(A^{n}\right)^{*}$ and $\operatorname{Ker}(f)=P$. Hence, $P$ is tight closed in a free $A$-module. Therefore every finitely generated projective $A$-module is tight closed in a free $R$-module.

Let $R$ be a ring. A submodule $K$ of an $R$-module $M$ is called closed [10, p. 548] if $K$ has no proper essential extension in $M$.

Proposition 3.4. Let $R$ be a ring and let $M$ be an $R$-module such that $Z(M)=$ 0 . If $N$ is tight closed in $M$, then $N$ is closed in $M$. 
Proof. Suppose that $N$ has a proper essential extension $E$ in $M$. Then there exists an element $e \in E \backslash N$. So, $0 \neq R e \subseteq E$. Since $N$ is essential in $E$, $\operatorname{Re} \cap N \neq 0$. There exists a non-zero element $n$ such that $n \in R e \cap N$. There exists an element $r \in R$ such that $n=r e$.

Now, let $f$ be any element in $G_{M^{*}}(N, 0)$. Then $n \in \operatorname{Ker}(f)$. So, $0=f(n)=$ $r f(e)$. Since $Z(M)=0$, we have $f(e)=0$. Hence $e \in \operatorname{Ker}(f)$. This shows that

$$
e \in \cap_{f \in G_{M} *(N, 0)} \operatorname{Ker}(f) .
$$

Since $N$ is tight closed in $M$, we have $e \in N$. This contradiction shows that $N$ has no proper essential extension.

Theorem 3.5. Let $R$ be a ring. Let $N$ be a submodule of an $R$-module $K$. If no proper extension of $N$ in $K$ is essential in $K$, then $N$ is essential in $K$.

Proof. Suppose that $N$ is not essential in $K$. Then there exists a non-zero submodule $L$ of $K$ such that $N \cap L=0$. By Zorn's lemma, we may assume that $L$ is maximal among such. By assumption, $N \oplus L$ is not essential in $K$. Then there exists a non-zero submodule $F$ of $K$ such that $(N \oplus L) \cap F=0$. Hence $N \cap(L \oplus F)=0$. By the maximality of $L, L=L \oplus F \supseteq F$, so $F=L \cap F=0$. This is a contradiction. Hence $N$ is essential in $K$.

Corollary 3.6. Let $R$ be a ring. Let $N$ be a submodule of $K$ and let $K$ be a submodule of $M$. If no proper extension of $N$ in $K$ is essential in $K$ and if $N$ is closed in $M$, then $N=K$.

Let $R$ be a ring and let $N$ be a submodule of $M$. Let

$$
K=\cap_{f \in G_{M^{*}}(N, 0)} \operatorname{Ker}(f) .
$$

Then $N \subseteq K$. If no proper extension of $N$ in $K$ is essential in $K$ and if $N$ is closed in $M$, then it follows from Corollary 3.6 that $N$ is tight closed in $M$.

It is well-known [4] that every epimorphism of a multiplication module onto itself is an automorphism. If $M$ is a non-zero multiplication $R$-module whose endomorphism ring is an integral domain, then we show that every non-zero endomorphism of $M$ is a monomorphism.

Lemma 3.7. Let $M$ be a non-zero multiplication $R$-module whose endomorphism ring $M^{*}$ is an integral domain. Then

(1) For every non-zero submodule $N$ of $M, G_{M^{*}}(N, 0)=0$. Hence every non-zero endomorphism of $M$ is a monomorphism.

(2) The only tight closed submodule of $M$ are 0 and $M$ itself.

Proof. (1) Suppose on the contrary that there exists a non-zero submodule $N$ of $M$ such that $G_{M^{*}}(N, 0) \neq 0$. Then there exists a non-zero $f$ in $G_{M^{*}}(N, 0)$. Since $M$ is a multiplication $R$-module, there exist ideals $I, J$ of $R$ such that $N=I M$ and $f(M)=J M$. Hence we have

$$
0=f(N)=f(I M)=I f(M)=I(J M)=(I J) M .
$$


This implies that $I J \subseteq \operatorname{Ann}_{R}(M)$. Since $M^{*}$ is an integral domain, it follows from the argument just prior to Example 1.1 that $\operatorname{Ann}_{R}(M)$ is a prime ideal of $R$. So, we have $I \subseteq \operatorname{Ann}_{R}(M)$ or $J \subseteq \operatorname{Ann}_{R}(M)$. If $I \subseteq \operatorname{Ann}_{R}(M)$, then $N=I M=0$, a contradiction. Or, if $J \subseteq \operatorname{Ann}_{R}(M)$, then $f(M)=J M=0$ and hence $f=0$, a contradiction. Therefore, for every non-zero submodule $N$ of $M, G_{M *}(N, 0)=0$.

Assume that there exists an non-zero endomorphism $f$ of $M$ such that $\operatorname{Ker}(f) \neq 0$. Then by the previous argument we have $f \in G_{M^{*}}(\operatorname{Ker}(f), 0)=0$. Thus $f=0$, a contradiction. Hence every non-zero endomorphism of $M$ is a monomorphism.

(2) Let $N$ be a non-zero tight closed submodule of $M$. Then by (1), $G_{M^{*}}(N, 0)=0$ and so $N=\cap_{f \in G_{M^{*}}(N, 0)} \operatorname{Ker}(f)=\operatorname{Ker}(0)=M$.

A submodule $L$ of an $R$-module $M$ is said to be $M$-cyclic if $L$ is isomorphic to $M / N$ for some submodule $N$ of $M$.

Let $L$ be a submodule of an $R$-module $M$. Assume that $L$ is $M$-cyclic. Then there exists a submodule $N$ of $M$ such that $L \cong M / N$. There exists an isomorphism $g: M / N \rightarrow L$. Consider the composite map

$$
f: M \stackrel{\pi}{\longrightarrow} M / N \stackrel{g}{\longrightarrow} L \stackrel{\text { inc }}{\longrightarrow} M .
$$

Then $f \in M^{*}$ and $f(M)=(\operatorname{inc} g \pi)(M)=L$.

Conversely, assume that there exists $f \in M^{*}$ such that $L=f(M)$. Then by the first isomorphism theorem for modules $L=f(M) \cong M / \operatorname{Ker}(f)$.

This shows that $L$ is $M$-cyclic if and only if there exists $f \in M^{*}$ such that $L=f(M)$.

An $R$-module $M$ is said to be semi-injective if every homomorphism from an $M$-cyclic submodule of $M$ to $M$ can be extended to $M$. Compare the following lemma with [13, Lemma 2.2].

Lemma 3.8. Let $R$ be a ring and let $M$ be an $R$-module. Then $M$ is semiinjective if and only if for every $f \in M^{*}, G_{M^{*}}(\operatorname{Ker}(f), 0)$ is a cyclic left ideal of $M^{*}$ generated by $f$.

Proof. Let $f$ be any element of $M^{*}$. For any $g \in M^{*}$,

$$
(g f)(\operatorname{Ker}(f))=g(f(\operatorname{Ker}(f)))=g(0)=0 .
$$

Hence $M^{*} f \subseteq G_{M^{*}}(\operatorname{Ker}(f), 0)$. Conversely, let $h \in G_{M^{*}}(\operatorname{Ker}(f), 0)$. Then $h(\operatorname{Ker}(f))=0$, so $\operatorname{Ker}(f) \subseteq \operatorname{Ker}(h)$. Define a map $\varphi: f(M) \rightarrow M$ by $\varphi(f(m))=h(m)$, where $m \in M$. Then

$$
f(m)=0 \Rightarrow m \in \operatorname{Ker}(f) \subseteq \operatorname{Ker}(h) \Rightarrow h(m)=0 .
$$


This shows that $\varphi$ is well-defined. Further, $\varphi$ is an $R$-homomorphism and $\varphi f=h$. Now, assume that $M$ is semi-injective. Consider the following diagram:

$$
\begin{array}{r}
0 \rightarrow f(M) \stackrel{\text { inc }}{\longrightarrow} M \\
\varphi \downarrow \\
M
\end{array}
$$

Then $f(M)$ is $M$-cyclic, so there exists $g \in M^{*}$ such that $g$ inc $=\varphi$. Hence

$$
h=\varphi f=g \operatorname{inc} f=g f .
$$

Thus $h \in M^{*} f$. This shows that $G_{M^{*}}(\operatorname{Ker}(f), 0) \subseteq M^{*} f$. Therefore

$$
G_{M^{*}}(\operatorname{Ker}(f), 0)=M^{*} f \text {. }
$$

Assume that for every $f \in M^{*}, G_{M^{*}}(\operatorname{Ker}(f), 0)$ is a cyclic left ideal of $M^{*}$ generated by $f$. Consider the following diagram:

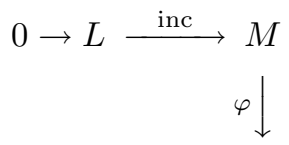

\section{$M$}

where $L$ is $M$-cyclic. Then there exists $f \in M^{*}$ such that $L=f(M) . \varphi f \in$ $M^{*}$ and $(\varphi f)(\operatorname{Ker}(f))=0$, so $\varphi f \in G_{M^{*}}(\operatorname{Ker}(f), 0)$. By our assumption, $G_{M^{*}}(\operatorname{Ker}(f), 0)=M^{*} f$. So, there exists $g \in M^{*}$ such that $\varphi f=g f$. Hence, for any $m \in M$,

$$
(g \text { inc })(f(m))=(g \operatorname{inc} f)(m)=(\varphi f)(m)=\varphi(f(m)) .
$$

This shows that ginc $=\varphi$. Therefore $M$ is semi-injective.

Lemma 3.9. Let $R$ be a ring. Let $M$ be an $R$-module such that $Z\left(M^{*}\right)=0$. If $f$ and $g$ are elements of $M^{*}$ such that $f g=1_{M}$, then $g f=1_{M}$.

Proof. Assume $f g=1_{M}$. Then $g \neq 0$. Further, $\left(g f-1_{M}\right) g=0$. Hence $g f-1_{M}=0$ and thus $g f=1_{M}$.

Theorem 3.10. Let $R$ be a ring. Let $M$ be a multiplication $R$-module such that $Z\left(M^{*}\right)=0$. Then $M$ is semi-injective if and only if $M^{*}$ is a division ring.

Proof. Assume that $M$ is semi-injective. Let $f$ be any non-zero element of $M^{*}$. Then by Lemma $3.7(1), \operatorname{Ker}(f)=0$. So,

$$
M^{*} f=G_{M^{*}}(\operatorname{Ker}(f), 0)=G_{M^{*}}(0,0)=M^{*} .
$$

By Lemma 3.9, $f$ is an epimorphism. Therefore $M^{*}$ is a division ring.

Conversely, assume that $M^{*}$ is a division ring. Let $f$ be any non-zero element of $M^{*}$. Then $f$ is an automorphism. Hence

$$
G_{M^{*}}(\operatorname{Ker}(f), 0)=G_{M^{*}}(0,0)=M^{*}=(f) .
$$

By Lemma 3.8, $M$ is semi-injective. 
An $R$-module $M$ is said to be self-cogenerated if every submodule of $M$ is tight closed. If $M$ is a simple $R$-module, then by the statement just posterior to Proposition 3.3, $M$ is self-cogenerated.

Theorem 3.11. Let $R$ be a commutative ring with identity. Let $M$ be a multiplication $R$-module such that $Z\left(M^{*}\right)=0$. Then the following statements are equivalent.

(1) $M$ is self-cogenerated;

(2) For any non-zero $f \in M^{*}, f$ is an epimorphism;

(3) $M$ is simple.

Proof. We have already known that $M^{*}$ is a commutative ring with identity. Hence by our hypothesis $M^{*}$ is an integral domain.

$(1) \Rightarrow(2)$. Assume (1). Let $f$ be any non-zero element of $M^{*}$. Then $\operatorname{Im}(f)(\subseteq M)$ is tight closed. By Lemma $3.7(2), \operatorname{Im}(f)=M$. Hence $f$ is an epimorphism. Hence (2) follows. field.

$(2) \Rightarrow(3)$. Assume $(2)$. By the statement just prior to Lemma $3.7, M^{*}$ is a

Now, let $N$ be any non-zero submodule of $M$. Since $M$ is a multiplication $R$-module, there exists an ideal $I$ of $R$ such that $N=I M$. Then

$$
N=I M=\varphi_{I}(M)=\varphi(I)(M) .
$$

Hence $\varphi(I) \neq 0$. There exists an element $r \in I$ such that $\varphi(r) \neq 0 . \varphi_{r}=\varphi(r) \neq$ 0 . By our assumption, $\varphi_{r}$ has an inverse $\varphi_{r}^{-1}$ in $M^{*}$. Further, $\varphi_{r}^{-1} \in M^{*}$. By Lemma $1.4, N$ is fully invariant. So,

$$
M=\varphi_{r}^{-1} \varphi_{r}(M) \subseteq \varphi_{r}^{-1}\left(\varphi_{I}(M)\right)=\varphi_{r}^{-1}(N) \subseteq N .
$$

Hence $N=M$. Thus, $M$ is simple. Therefore (3) follows.

$(3) \Rightarrow(1)$. Assume (3). Let $N$ be any submodule of $M$. Then $N=0$ or $N=M$. By the statement just posterior to Proposition 3.3, 0 and $M$ are tight closed. Hence $N$ is tight closed. Thus $M$ is self-cogenerated. Therefore, (1) follows.

Corollary 3.12. Let $R$ be a commutative ring with identity. Let $M$ be a multiplication $R$-module such that $Z\left(M^{*}\right)=0$. Then the following statements are equivalent.

(1) $M$ is semi-injective;

(2) $M^{*}$ is a field;

(3) $M$ is self-cogenerated;

(4) For any non-zero $f \in M^{*}, f$ is an epimorphism;

(5) $M$ is simple.

Proof. $M^{*}$ is a commutative ring with identity.

$(5) \Rightarrow(2)$ follows from Schur's Lemma. (2) $\Rightarrow(5)$ follows from the proof of Theorem $3.11(2) \Rightarrow(3)$. The remainder of the proof follows from Theorem 3.10 and Theorem 3.11 . 


\section{References}

[1] S.-S. Bae, On submodules inducing prime ideals of endomorphism ring, East Asian Math. J. 16 (2000), no. 1, 33-48.

[2] _ Modules with prime endomorphism rings, J. Korean Math. Soc. 38 (2001), no. 5, 987-1030.

[3] C. W. Choi, Multiplication modules and endomorphisms, Math. J. Toyama Univ. 18 (1995), 1-8.

[4] C. W. Choi and P. F. Smith, On endomorphisms of multiplication modules, J. Korean Math. Soc. 31 (1994), no. 1, 89-95.

[5] Z. A. El-Bast and P. F. Smith, Multiplication modules, Comm. Algebra 16 (1988), no. 4, 755-779.

[6] E. S. Kim and C. W. Choi, On multiplication modules, Kyungpook Math. J. 32 (1992), no. $1,97-102$

[7] S. C. Lee, Finitely generated modules, J. Korean Math. Soc. 28 (1991), no. 1, 1-11.

[8] H. Matsumura, Commutative Ring Theory, Cambridge University Press, Cambridge, 1989.

[9] S. Mandal, Projective Modules and Complete Intersections, Springer-Verlag, Berlin, 1997.

[10] E. Mermut, C. Santa-Clara, and P. F. Smith, Injectivity relative to closed submodules, J. Algebra 321 (2009), no. 2, 548-557.

[11] D. W. Sharpe and P. Vámos, Injective Modules, Cambridge University Press, LondonNew York, 1972.

[12] W. Vasconcelos, On finitely generated flat modules, Trans. Amer. Math. Soc. 138 (1969), 505-512.

[13] S. Wongwai, On the endomorphism ring of a semi-injective module, Acta Math. Univ. Comenian. (N.S.) 71 (2002), no. 1, 27-33.

Department of Mathematics Education

Chonbuk National University

Chonju 561-756, Korea

AND

Department of Mathematics

The University of COlorado AT Boulder

395 UCB

Boulder, Colorado 80309-0395, USA

E-mail address: scl@chonbuk.ac.kr; Sang.C.Lee@Colorado.EDU 